\title{
Highly Conjugated Graphitic Carbon Nitride Nanofoam for Photocatalytic Hydrogen Evolution
}

Chuan-Qi Cheng ${ }^{\dagger}$, Yi Feng ${ }^{\dagger}$, Zi-Zheng Shił, Yun-Long Zhou ${ }^{\dagger}$, Wen-Jing Kang ${ }^{\dagger}$, Zhe

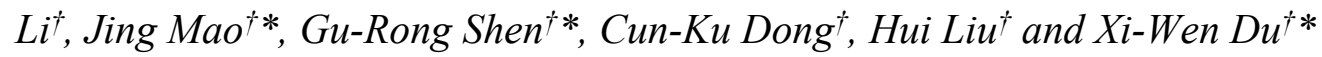

$\dagger$ Institute of New Energy Materials, School of Materials Science and Engineering, Tianjin University, Tianjin 300072, China

\$ School of Chemistry and Materials, Longyan University, Longyan City, Fujian Province, 364012, China 


\section{Supplementary Figures}

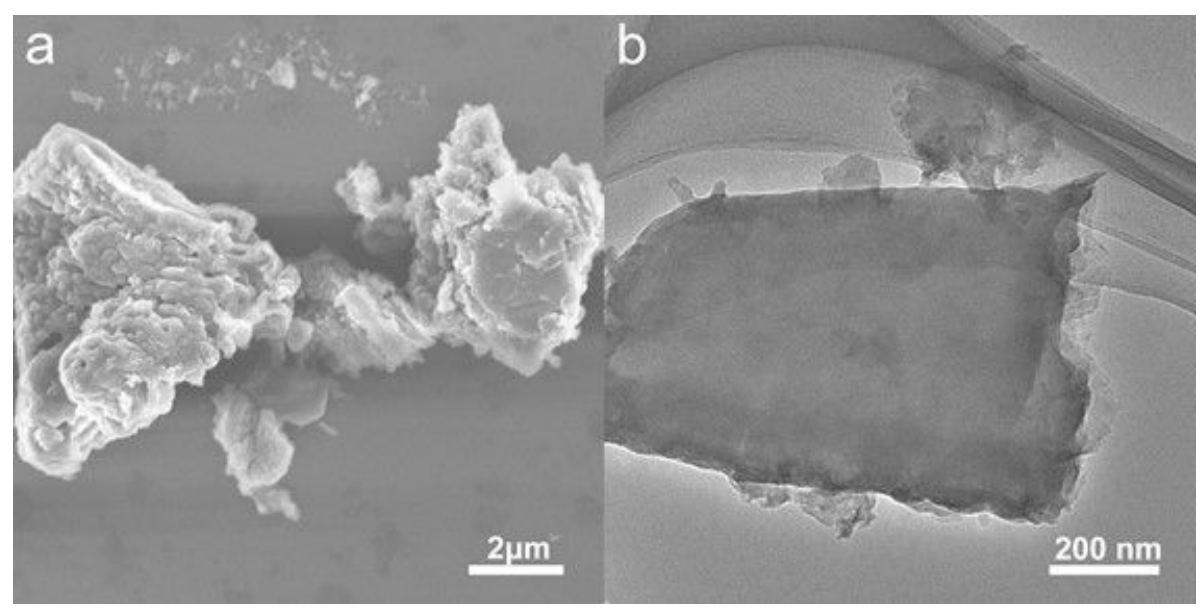

Figure S1. SEM image (a) and TEM image (b) of bulk g-CN.

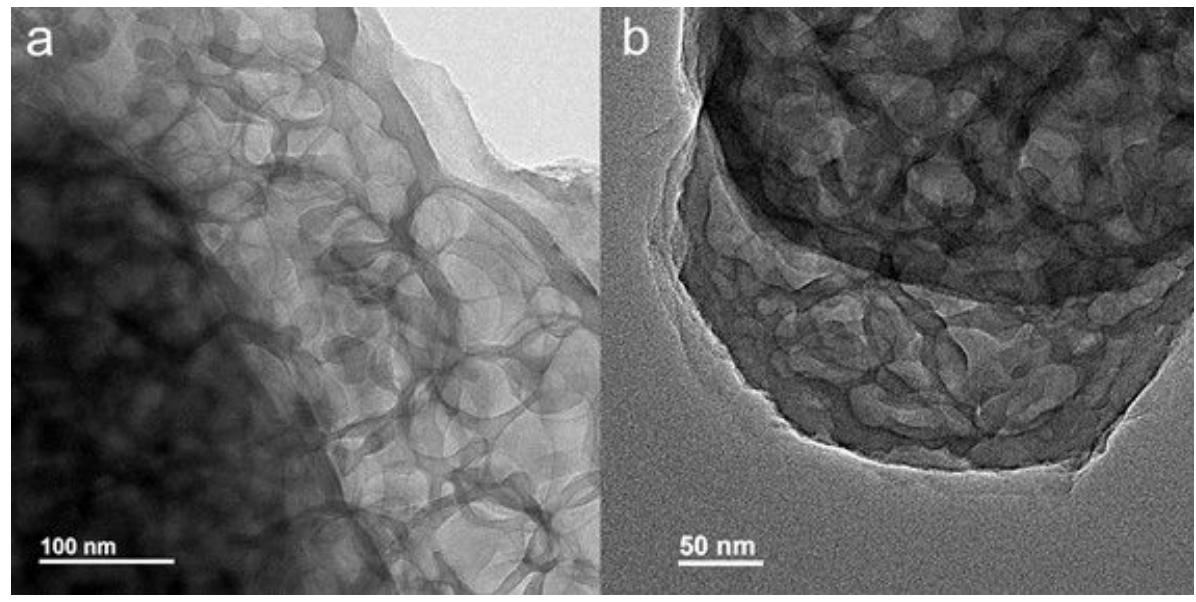

Figure S2. TEM images of the intermediates of (a) nanosheet and (b) nanofoam. 


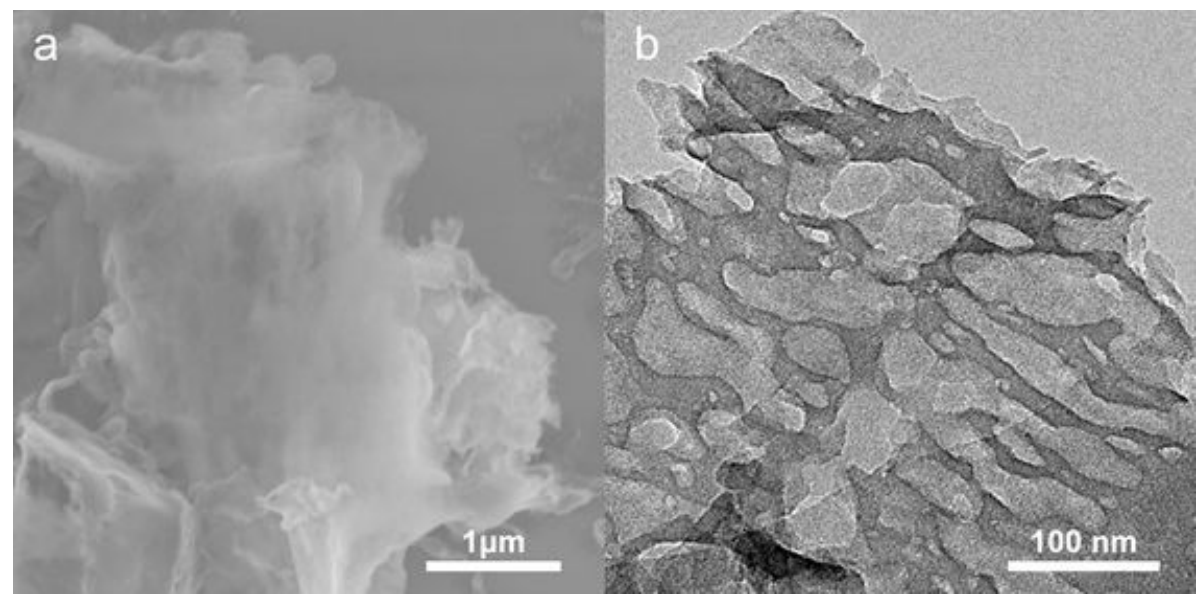

Figure S3. SEM image (a) and TEM image (b) of nanosheet.

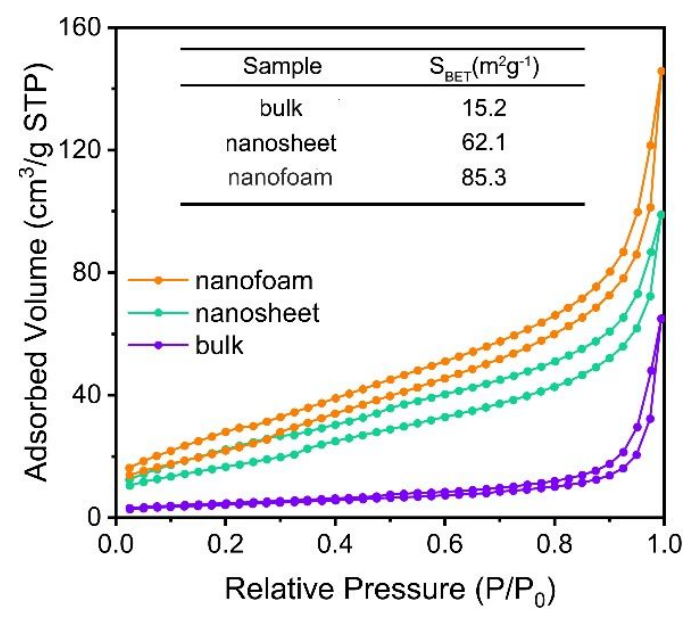

Figure S4. $\mathrm{N}_{2}$ sorption isotherms of bulk, nanosheet and nanofoam samples. 

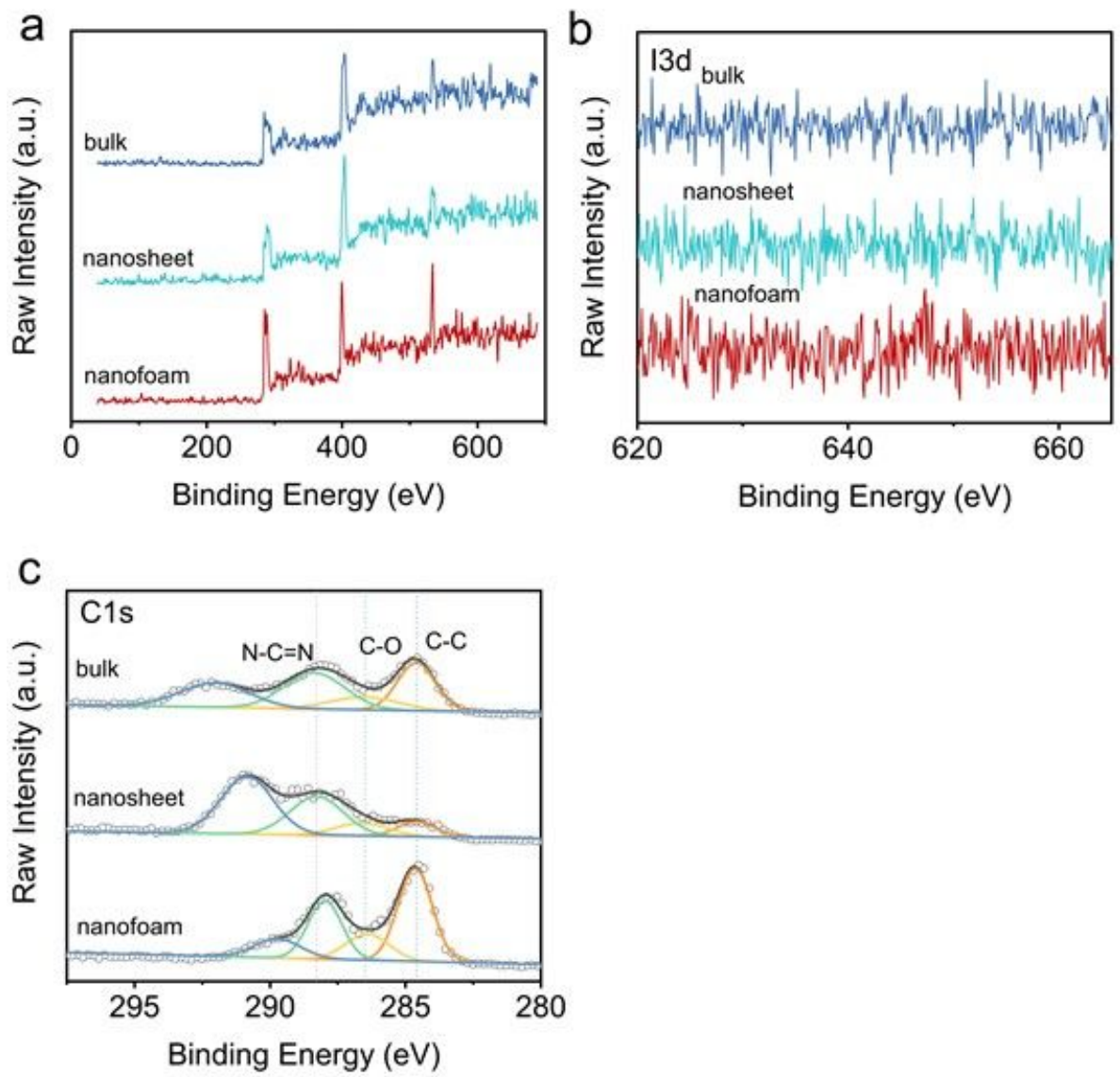

Figure S5. (a) Survey SRPES spectra, (b) high-resolution SRPES spectra of I 3d and (c) high-resolution SRPES spectra of C 1s for bulk, nanosheet and nanofoam samples.

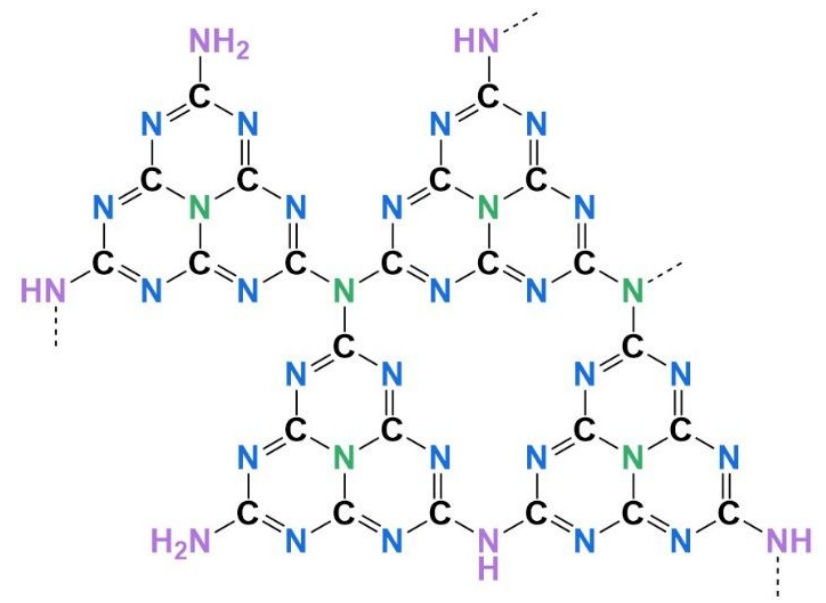

Figure S6. Molecular model of $\mathrm{g}-\mathrm{CN}$, where $\mathrm{N}$ atoms in $\mathrm{C}-\mathrm{N}=\mathrm{C}$ bonds, framework $\mathrm{N}-(\mathrm{C})_{3}$ bonds, and the unpolymerized $\mathrm{C}-\mathrm{NH}_{\mathrm{x}}$ bonds are labelled with blue, green and purple colors 

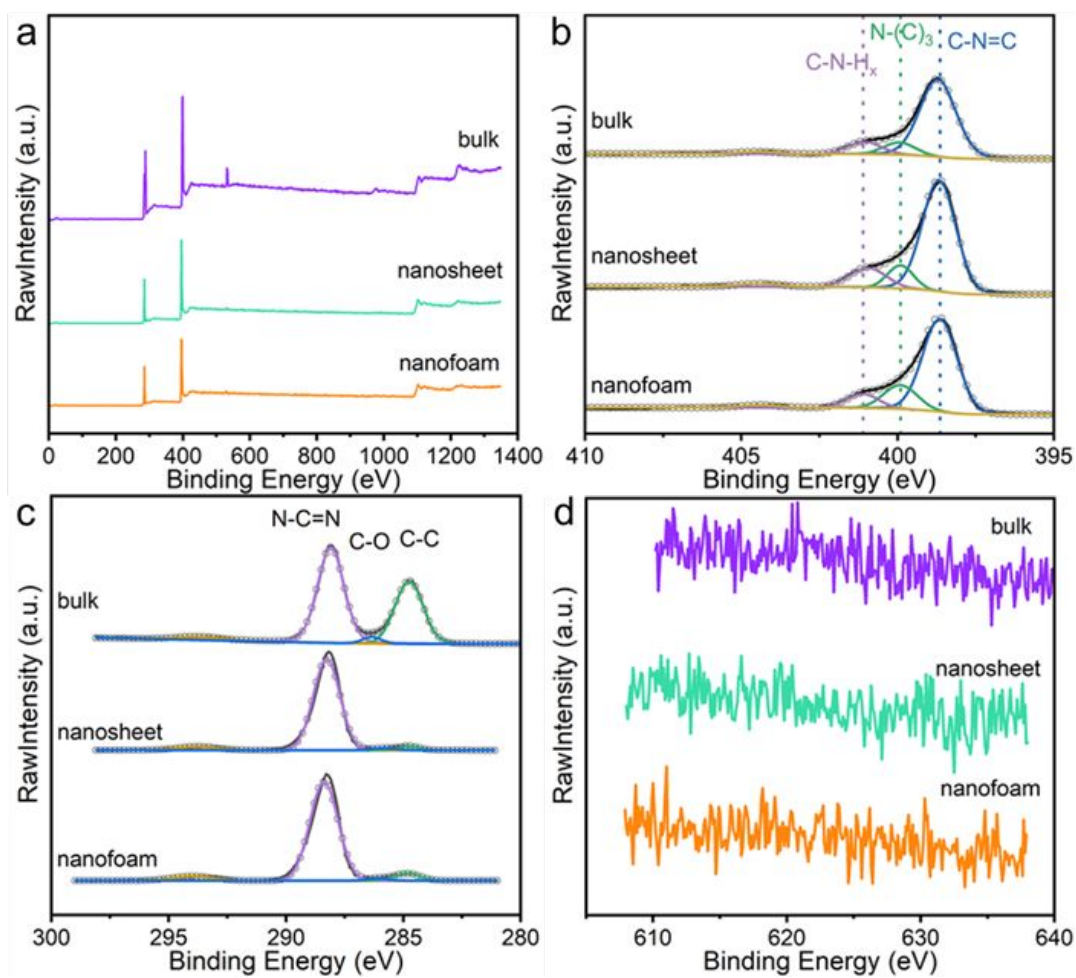

Figure S7. (a) Survey XPS spectra, and high-resolution XPS of (b) N 1s, (c) C 1s and (d) I 3d for bulk, nanosheet and nanofoam samples.

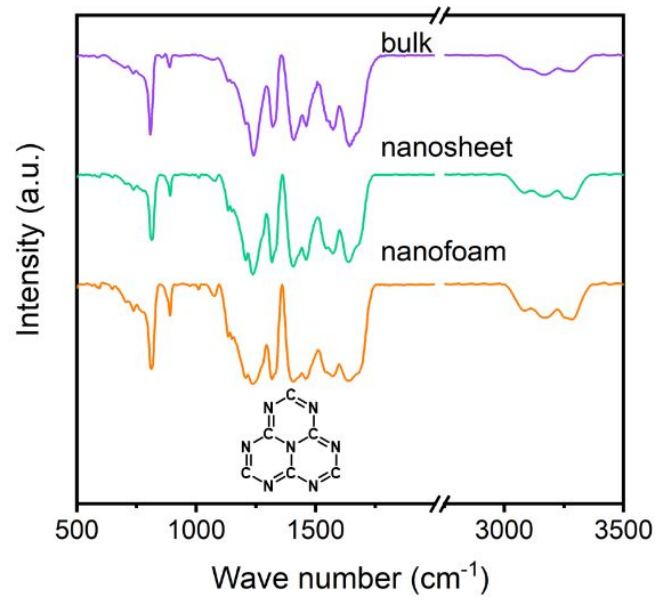

Figure S8. FTIR spectra for bulk, nanosheet and nanofoam samples. 


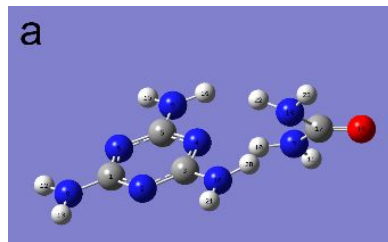

Initial State

d

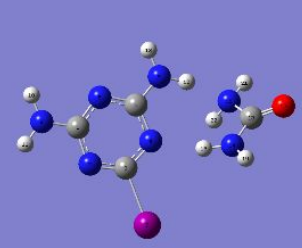

Initial State

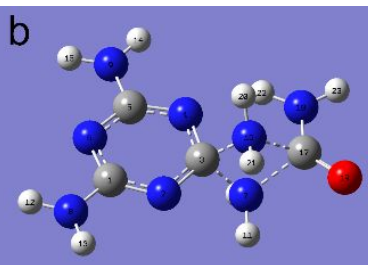

Transition State

e

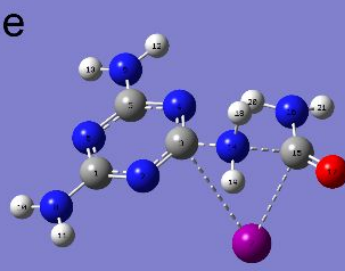

Transition State

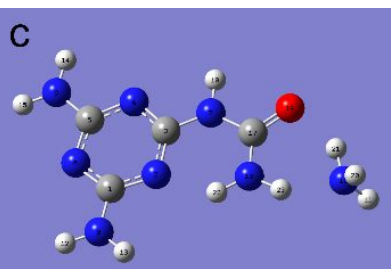

Final State

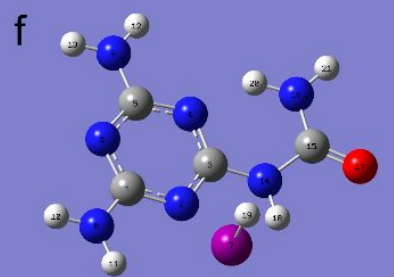

Final State

Figure S9. A computational model for the polycondensation of nanosheet and nanofoam, where $\mathrm{H}, \mathrm{C}, \mathrm{N}, \mathrm{O}, \mathrm{I}$ atoms are distinguished by (white), C (grey), N (blue), O (red), I (purple) color.

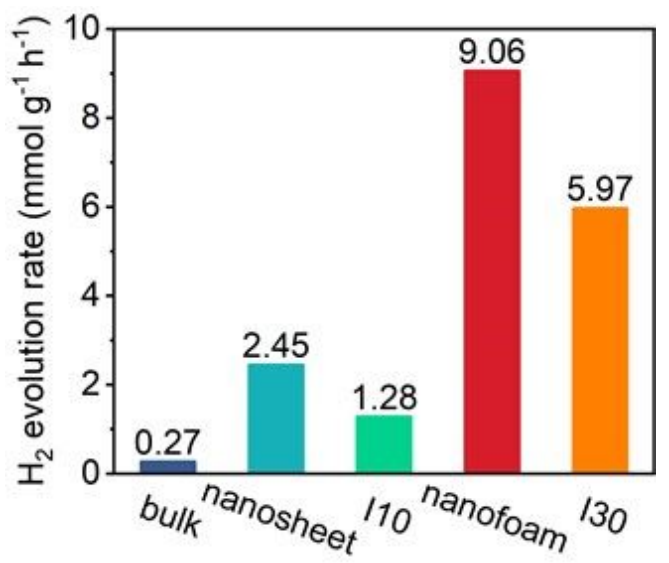

Figure S10. $\mathrm{H}_{2}$ production rates for bulk, nanosheet and nanofoam samples (The I10 and I30 samples are prepared by using $10 \mathrm{~mL}$ and $30 \mathrm{~mL}$ hydroiodic acid solution replaces the intermediates.) 


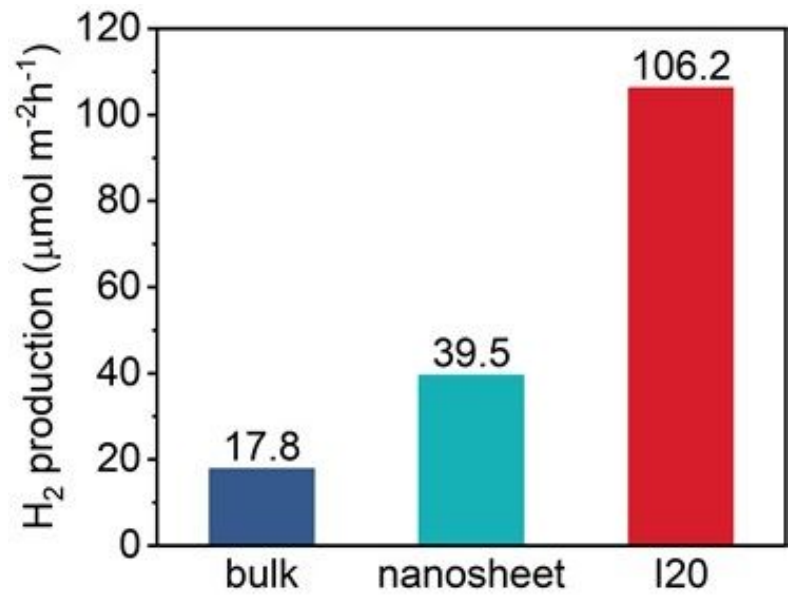

Figure $\mathbf{S 1 1 ~} \mathrm{H}_{2}$ production rates for bulk, nanosheet and nanofoam samples normalized by specific surface area.
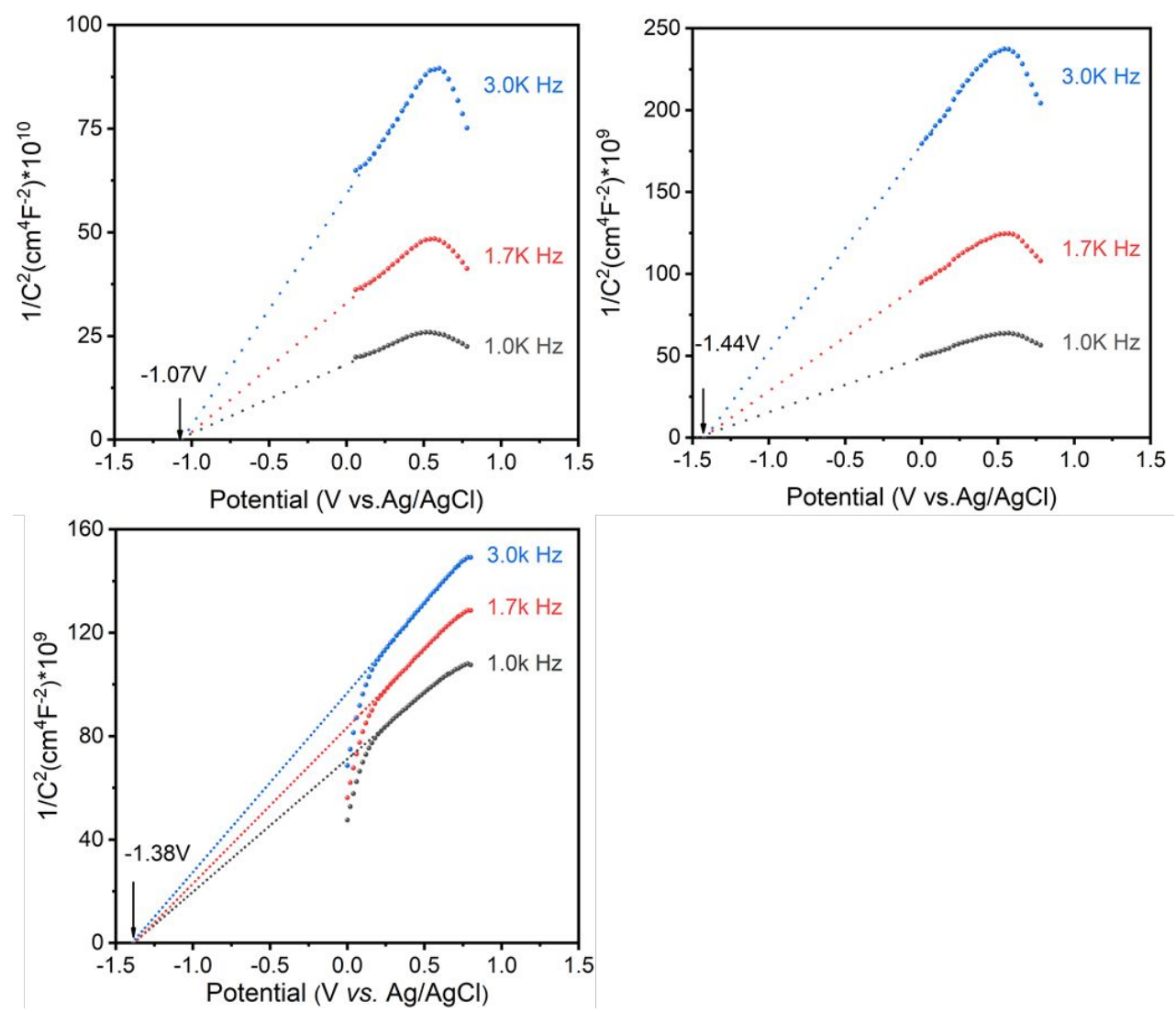

Figure S12. Mott-Schottky plots of(a) bulk, (b) nanosheet and (c) nanofoam. 


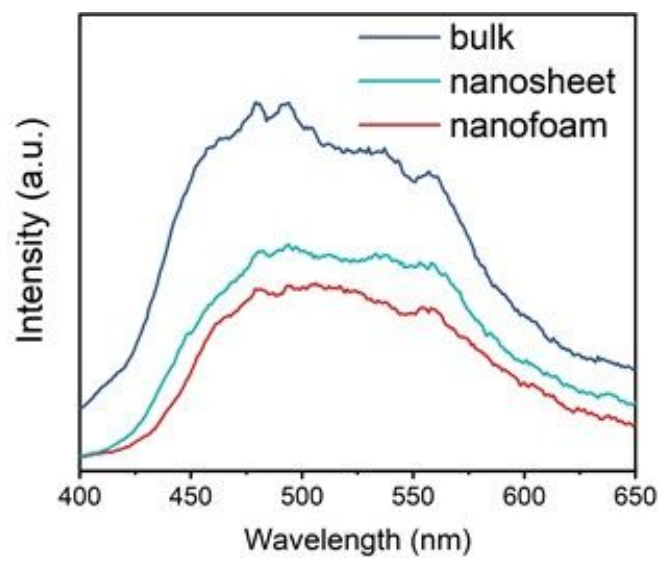

Figure S13. photoluminescence spectra of bulk, nanosheet and nanofoam samples.

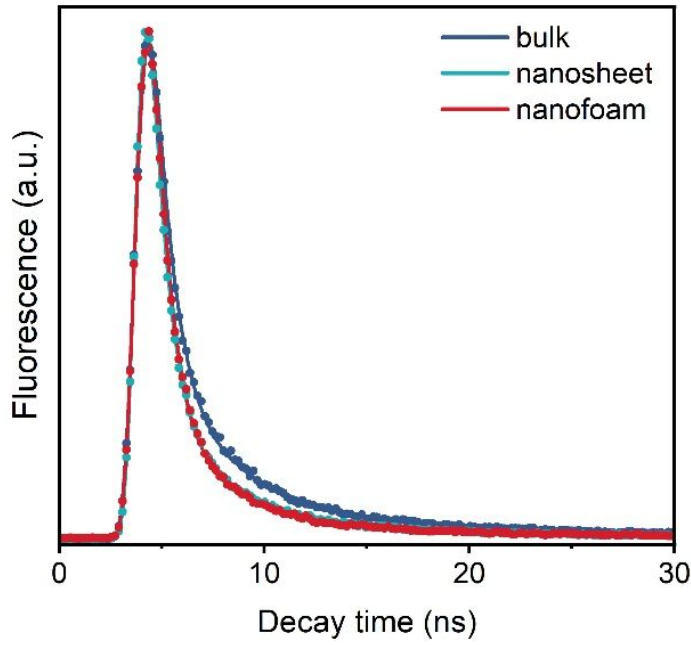

Figure S14. Time-resolved emission spectroscopy (TRES) and fitting curves of bulk, nanosheet and nanofoam. 


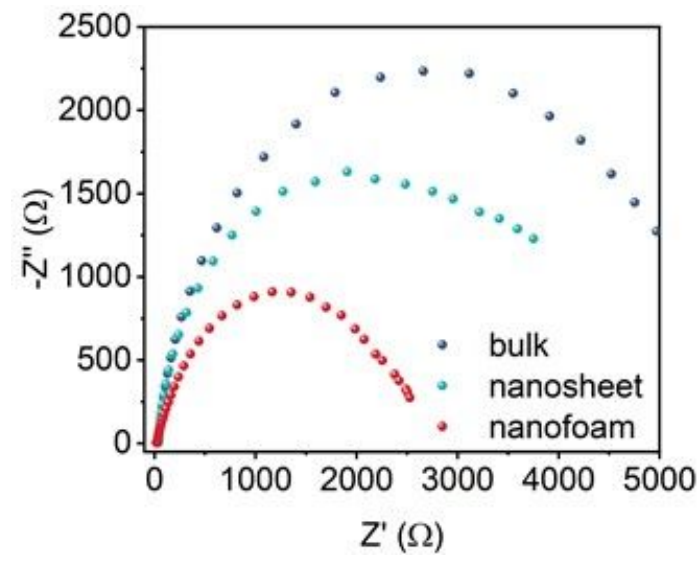

Figure S15 EIS Nyquist plots of bulk, nanosheet and nanofoam samples.

a

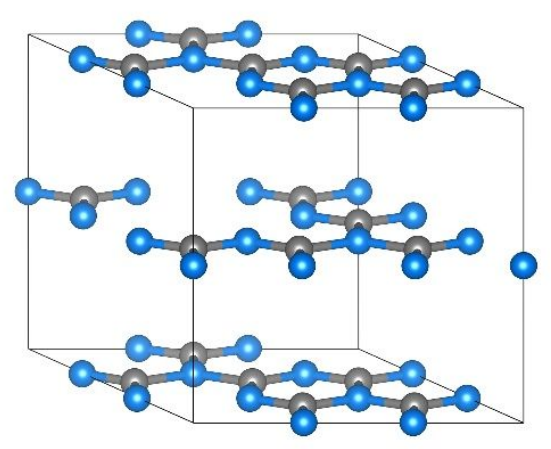

b

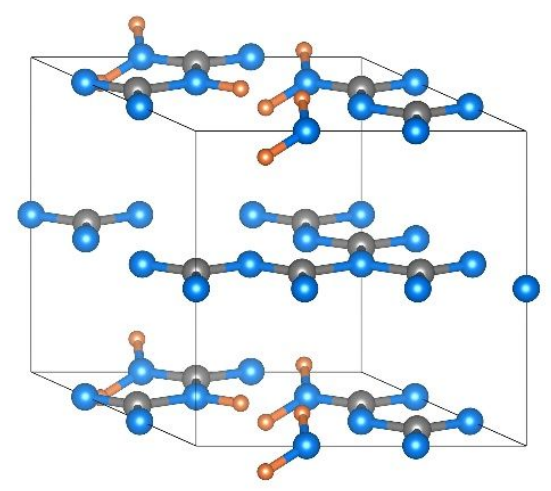

Figure S16. The atomic models of perfect $\mathrm{g}-\mathrm{CN}(\mathrm{a})$ and the $\mathrm{g}-\mathrm{CN}$ with $-\mathrm{NH}_{\mathrm{x}}$ defects

(b) 


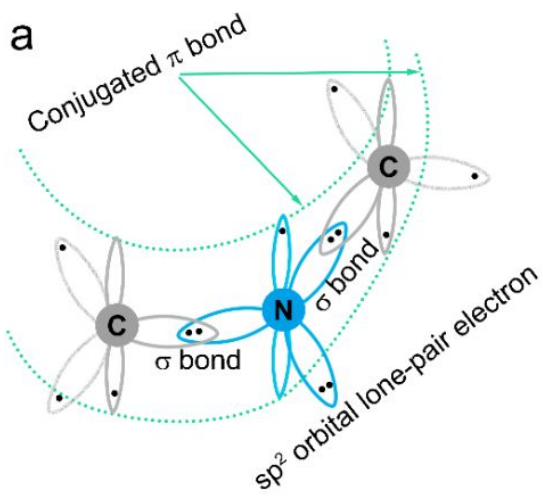

$\mathrm{N}$ from $\mathrm{C}-\mathrm{N}=\mathrm{C}$ site b

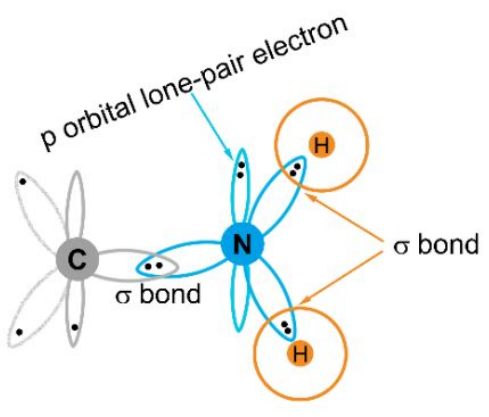

$\mathrm{N}$ from C- $\mathrm{NH}_{2}$ site

Figure S17. Schematic diagram of orbital hybridization and bonding at $\mathrm{C}-\mathrm{N}=\mathrm{C}$ site and C-NH 2 site
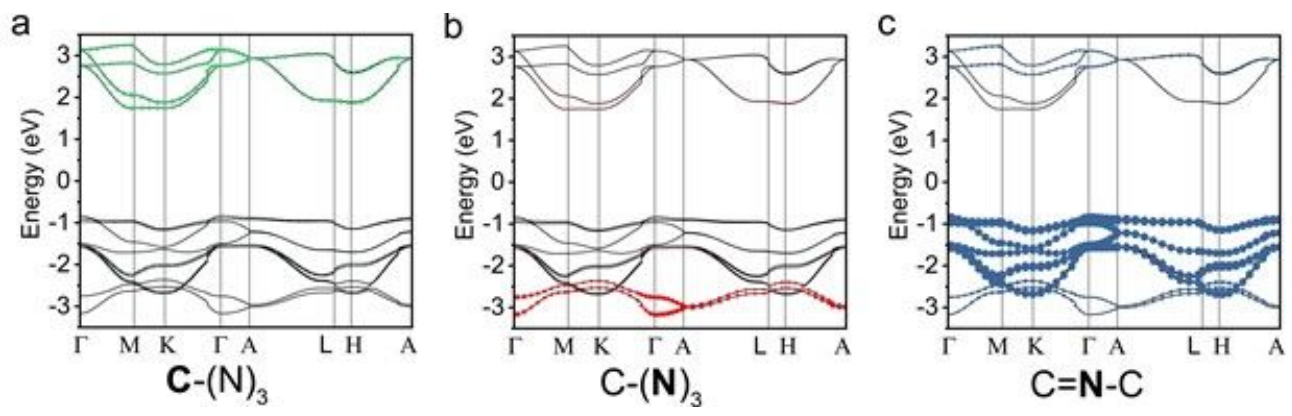

Figure S18. The projected band structures of perfect g-CN 

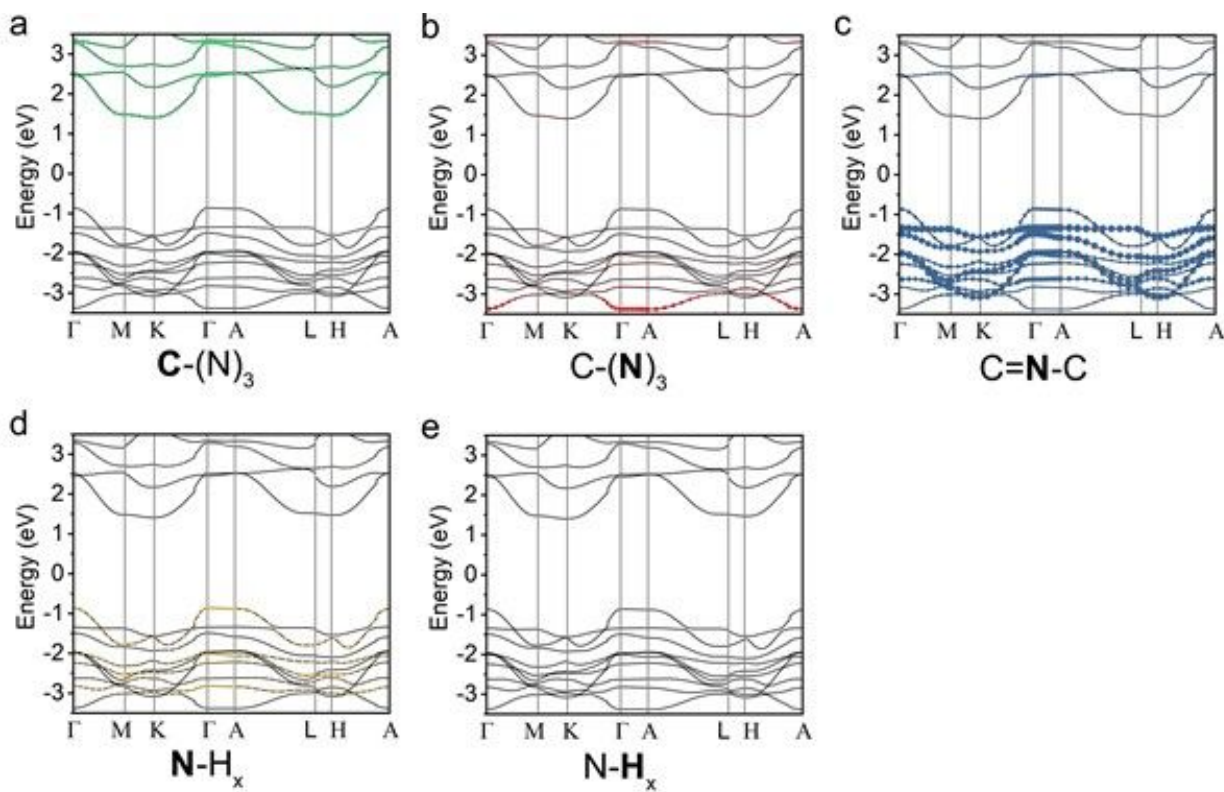

Figure S19. The projected band structures of the g-CN with $-\mathrm{NH}_{\mathrm{x}}$ defect
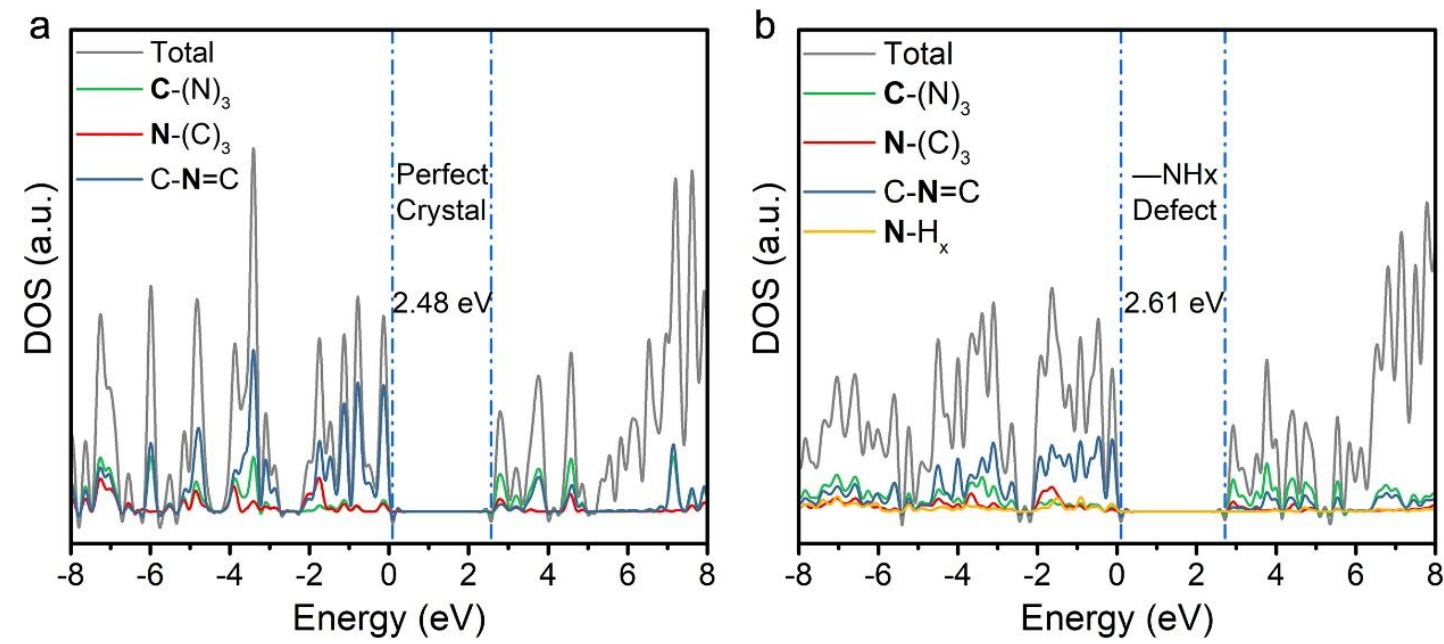

Figure S20. The local density of states (LDOS) of the perfect g-CN and the g-CN with

$-\mathrm{NH}_{\mathrm{x}}$ defect. 


\section{Supplementary Tables}

Table S1. Proportions of different N sites according to SRPES results

\begin{tabular}{cccc}
\hline samples & $\mathbf{C}-\mathbf{N}=\mathbf{C}$ & $\mathbf{N}-(\mathbf{C})_{3}$ & $\mathbf{C}-\mathbf{N H}_{\mathbf{x}}$ \\
\hline bulk & $35.4 \%$ & $26.7 \%$ & $37.9 \%$ \\
nanosheet & $21.0 \%$ & $19.8 \%$ & $59.2 \%$ \\
nanofoam & $43.0 \%$ & $28.6 \%$ & $28.4 \%$ \\
\hline
\end{tabular}

Table S2. Proportions of different N sites according to XPS results

\begin{tabular}{cccc}
\hline samples & $\mathbf{C}-\mathbf{N}=\mathbf{C}$ & $\mathbf{N}-(\mathbf{C})_{3}$ & $\mathbf{C}-\mathbf{N H}_{\mathbf{x}}$ \\
\hline bulk & $78.5 \%$ & $10.4 \%$ & $11.1 \%$ \\
nanosheet & $74.7 \%$ & $11.5 \%$ & $13.8 \%$ \\
nanofoam & $72.5 \%$ & $17.6 \%$ & $9.9 \%$ \\
\hline
\end{tabular}

Table S3. Atomic ratio of the elements obtained according to XPS

\begin{tabular}{ccccc}
\hline samples & $\mathbf{C}$ & $\mathbf{N}$ & $\mathbf{O}$ & $\mathbf{C} / \mathbf{N}$ \\
\hline bulk & $53.21 \%$ & $42.24 \%$ & $4.55 \%$ & $5: 4$ \\
nanosheet & $41.78 \%$ & $56.92 \%$ & $1.30 \%$ & $3: 4$ \\
nanofoam & $41.82 \%$ & $56.22 \%$ & $1.96 \%$ & $3: 4$ \\
I10 & $41.94 \%$ & $56.69 \%$ & $1.37 \%$ & $3: 4$ \\
I30 & $42.2 \%$ & $56.48 \%$ & $1.33 \%$ & $3: 4$ \\
\hline
\end{tabular}


Table S4. Best fitted parameters of time resolved emission spectra (Figure S14)

\begin{tabular}{ccccccccccc}
\hline samples & \multicolumn{2}{c}{$\begin{array}{c}\text { Decay life times } \\
\text { (ns) }\end{array}$} & \multicolumn{3}{c}{$\begin{array}{c}\text { Fraction } \\
\text { contribution }\end{array}$} & Goodness of fit & $\begin{array}{c}\text { Average } \\
\text { life time }\end{array}$ \\
& & $\tau_{1}$ & $\tau_{2}$ & $\tau_{3}$ & $\mathrm{f}_{1}$ & $\mathrm{f}_{2}$ & $\mathrm{f}_{3}$ & parameter $\left(\chi^{2}\right)$ & (ns) \\
\hline bulk & 1.13 & 5.44 & 33.19 & 82 & 16 & 1 & 1.37 & 2.24 \\
nanosheet & 0.97 & 4.49 & 27.52 & 86 & 13 & 1 & 1.23 & 1.64 \\
nanofoam & 0.93 & 4.05 & 24.93 & 86 & 13 & 1 & 1.08 & 1.53 \\
\hline
\end{tabular}


Table S5. Performance comparison of various g-CN photocatalysts reported in literature

\begin{tabular}{|c|c|c|c|c|c|}
\hline Photocatalysts & $\begin{array}{l}\text { Light } \\
\text { source }\end{array}$ & $\begin{array}{l}\text { Reaction } \\
\text { conditions }\end{array}$ & $\begin{array}{l}\text { Hydrogen } \\
\text { evolution rate } \\
(\mu \mathrm{mol} / \mathrm{g} / \mathrm{h})\end{array}$ & AQY & Ref. \\
\hline nanofoam & $\begin{array}{l}300 \mathrm{~W} \mathrm{Xe} \\
\text { lamp, } \lambda> \\
420 \mathrm{~nm}\end{array}$ & $\begin{array}{l}3 \text { wt. } \% \text { Pt, TEOA } \\
(20 \text { vol } \%)\end{array}$ & 9066 & $\begin{array}{l}18.9 \% \\
(420 \\
\mathrm{nm})\end{array}$ & $\begin{array}{l}\text { This } \\
\text { work }\end{array}$ \\
\hline CN-ATZ-NaK & $\begin{array}{l}\text { White } \\
\text { LED light } \\
(50 \mathrm{~W} \lambda> \\
420 \mathrm{~nm})\end{array}$ & $\begin{array}{l}3 \text { wt. } \% \mathrm{Pt} \text {, TEOA } \\
(10 \\
\text { vol } \%), 0.01 \mathrm{molK}_{2} \mathrm{H} \\
\mathrm{PO}_{4}\end{array}$ & 13060 & $\begin{array}{l}65 \% \\
(420 \\
\mathrm{nm})\end{array}$ & 1 \\
\hline $\mathrm{CNPS}_{-} \mathrm{NH}_{2}$ & $\begin{array}{l}300 \mathrm{~W} \mathrm{Xe} \\
\text { lamp, } \lambda> \\
420 \mathrm{~nm}\end{array}$ & $\begin{array}{l}3 \text { wt. } \% \text { Pt, TEOA } \\
(20 \mathrm{vol} \%)\end{array}$ & 3761 & N/A & 2 \\
\hline $\begin{array}{l}\text { Porous Few- } \\
\text { Layer } \mathrm{g}-\mathrm{C}_{3} \mathrm{~N}_{4}\end{array}$ & $\begin{array}{l}300 \mathrm{~W} \mathrm{Xe} \\
\text { lamp, } \lambda> \\
420 \mathrm{~nm}\end{array}$ & $\begin{array}{l}1 \text { wt. } \% \text { Pt, TEOA } \\
(20 \mathrm{vol} \%)\end{array}$ & 7990 & $\begin{array}{l}9.8 \% \\
(420 \\
\mathrm{nm})\end{array}$ & 3 \\
\hline $\begin{array}{l}\text { horn-like g- } \\
\mathrm{C}_{3} \mathrm{~N}_{4} \text { tube }\end{array}$ & $\begin{array}{l}300 \mathrm{~W} \text { Xe } \\
\text { lamp, } \lambda> \\
420 \mathrm{~nm}\end{array}$ & $\begin{array}{l}1 \text { wt.\% Pt, Lactic } \\
\text { acid (10 vol\%) }\end{array}$ & 1354 & $\begin{array}{l}14.3 \% \\
(420 \\
\mathrm{nm})\end{array}$ & 4 \\
\hline
\end{tabular}




\begin{tabular}{|c|c|c|c|c|c|}
\hline $\begin{array}{l}\text { Ultrathin } \\
\text { porous N/g- } \\
\mathrm{C}_{3} \mathrm{~N}_{4}\end{array}$ & $\begin{array}{l}300 \mathrm{~W} \text { Xe } \\
\text { lamp, } \lambda> \\
420 \mathrm{~nm}\end{array}$ & $\begin{array}{l}1 \text { wt. } \% \text { Pt, Lactic } \\
\text { acid (20 vol\%) }\end{array}$ & 3579 & $\begin{array}{l}27.8 \% \\
(420 \\
\mathrm{nm})\end{array}$ & 5 \\
\hline $\begin{array}{l}\text { Atomically } \\
\text { thin } \quad \mathrm{g}-\mathrm{C}_{3} \mathrm{~N}_{4} \\
\text { nanomesh }\end{array}$ & $\begin{array}{l}300 \mathrm{~W} \text { Xe } \\
\text { lamp, } \lambda> \\
420 \mathrm{~nm}\end{array}$ & $\begin{array}{l}3 \text { wt. } \% \text { Pt, TEOA } \\
(10 \text { vol } \%)\end{array}$ & 8510 & $\begin{array}{l}5.1 \% \\
(420 \\
\mathrm{nm})\end{array}$ & 6 \\
\hline CCTs & $\begin{array}{l}300 \mathrm{~W} \text { Xe } \\
\text { lamp, } \lambda> \\
400 \mathrm{~nm}\end{array}$ & $\begin{array}{l}3 \text { wt. } \% \text { Pt, methanol } \\
(25 \text { vol } \%)\end{array}$ & 3538.3 & $\begin{array}{l}10.9 \% \\
(420 \\
\mathrm{nm})\end{array}$ & 7 \\
\hline $\begin{array}{l}\mathrm{g}_{-} \mathrm{C}_{3} \mathrm{~N}_{4} \quad(550) \\
\text { "seaweed" }\end{array}$ & $\begin{array}{l}300 \mathrm{~W} \text { Xe } \\
\text { lamp, } \lambda> \\
420 \mathrm{~nm}\end{array}$ & $\begin{array}{l}3 \text { wt. } \% \text { Pt, TEOA } \\
(10 \text { vol } \%)\end{array}$ & 9900 & $\begin{array}{l}7.8 \% \\
(420 \\
\mathrm{nm})\end{array}$ & 8 \\
\hline $\begin{array}{l}\text { PCN-U-AC } \\
\text { Nanosheet }\end{array}$ & $\begin{array}{l}300 \mathrm{~W} \text { Xe } \\
\text { lamp, } \lambda> \\
400 \mathrm{~nm}\end{array}$ & $\begin{array}{l}3 \text { wt. } \% \text { Pt, TEOA } \\
(9.1 \text { vol } \%)\end{array}$ & 5222 & $\begin{array}{l}11.3 \% \\
(405 \\
\mathrm{nm})\end{array}$ & 9 \\
\hline
\end{tabular}




\section{References}

1. Zhang, G. et al. Tailoring the Grain Boundary Chemistry of Polymeric Carbon Nitride for Enhanced Solar Hydrogen Production and CO 2 Reduction. Angew. Chemie - Int. Ed. 58, 3433-3437 (2019).

2. Meng, N. et al. Engineering oxygen-containing and amino groups into twodimensional atomically-thin porous polymeric carbon nitrogen for enhanced photocatalytic hydrogen production. Energy Environ. Sci. 11, 566-571 (2018).

3. Xiao, Y. et al. Molecule Self-Assembly Synthesis of Porous Few-Layer Carbon Nitride for Highly Efficient Photoredox Catalysis. J. Am. Chem. Soc. 141, 2508-2515 (2019).

4. Liu, C. et al. Intermediate-mediated strategy to horn-like hollow mesoporous ultrathin g-C3N4tube with spatial anisotropic charge separation for superior photocatalytic $\mathrm{H}_{2}$ evolution. Nano Energy 41, 738-748 (2017).

5. Tian, N. et al. Precursor-reforming protocol to 3D mesoporous gC3N4established by ultrathin self-doped nanosheets for superior hydrogen evolution. Nano Energy 38, 72-81 (2017).

6. Han, Q. et al. Atomically Thin Mesoporous Nanomesh of Graphitic $\mathrm{C}_{3} \mathrm{~N}_{4}$ for High-Efficiency Photocatalytic Hydrogen Evolution. ACS Nano (2016)

7. Wang, Y. et al. Carbon Quantum Dot Implanted Graphite Carbon Nitride Nanotubes: Excellent Charge Separation and Enhanced Photocatalytic Hydrogen Evolution. Angew. Chemie - Int. Ed. 57, 5765-5771 (2018).

8. Han, Q., Wang, B., Zhao, Y., Hu, C. \& Qu, L. A Graphitic-C3N4 'seaweed' 
Architecture for Enhanced Hydrogen Evolution. Angew. Chemie - Int. Ed. 54, 11433-11437 (2015).

9. Yang, P., Ou, H., Fang, Y. \& Wang, X. A Facile Steam Reforming Strategy to Delaminate Layered Carbon Nitride Semiconductors for Photoredox Catalysis. Angew. Chemie - Int. Ed. 56, 3992-3996 (2017). 